\title{
HIV testing among Portuguese men who have sex with men - results from the European MSM Internet Survey (EMIS)
}

\author{
C Carvalho, ${ }^{1,2}$ R Fuertes, ${ }^{3}$ R Lucas, ${ }^{1,2}$ A Martins, ${ }^{1,2}$ MJ Campos, ${ }^{3}$ L Mendão, ${ }^{3}$ AJ Schmidt ${ }^{4}$ and H Barros ${ }^{1,2}$ \\ ${ }^{1}$ Department of Clinical Epidemiology, Predictive Medicine and Public Health, University of Porto Medical School, Porto, \\ Portugal, ${ }^{2}$ Institute of Public Health, University of Porto, Porto, Portugal, ${ }^{3}$ Grupo Português de Activistas sobre \\ Tratamentos VIH/SIDA (GAT), Lisboa, Portugal and ${ }^{4}$ Sigma Research, Department of Social \& Environmental Health \\ Research, London School of Hygiene and Tropical Medicine, London, UK
}

Objectives

To describe HIV testing behaviour and context of MSM in Portugal participating in the European MSM Internet Survey (EMIS).

Methods

Data for the Portuguese sample were extracted and those for 5187 participants were analysed. Multivariate logistic regression models were fitted to quantify the association between participants' characteristics and HIV testing behaviour and context.

Results

Seventy-two percent of the participants had ever been tested for HIV and among those ever tested, 11\% were diagnosed with HIV. Primary care was the most common testing setting for HIV-negative men (37\%). Compared to those never tested, men who had ever taken an HIV test had higher educational level (aOR 1.89, 95\% CI 1.67-2.14) and identified themselves as gay/homosexual more frequently (aOR 1.94 , 95\% CI 1.70-2.20). HIV testing odds significantly increased with the number of sexual partners in the previous 12 months. Those who reported unprotected anal intercourse (UAI) with a partner of unknown or serodiscordant HIV status in the previous 12 months were less likely to report an HIV test (aOR 0.38, 95\% CI 0.33-0.44). Among those never tested or who tested negative, $41 \%$ and $22 \%$ reported UAI with a partner of unknown or serodiscordant status in the previous 12 months, respectively. Among men with diagnosed HIV, 72\% were currently on antiretroviral therapy and 58\% reported an undetectable viral load. More than one third (38\%) of those who had detectable or unknown/undisclosed viral load reported at least one episode of UAI with a partner of unknown or serodiscordant HIV status in the last 12 months.

\section{Conclusions}

Actual interventions should focus on: improving testing uptake and counselling; increasing treatment coverage; achieving and maintaining an undetectable viral load; and intensifying prevention efforts focused on consistent condom use.

Keywords: HIV, HIV Testing, Men who have sex with men, Web-based survey, Behavioral Surveillance

Accepted 12 June 2013

\section{Introduction}

The European HIV epidemic is largely concentrated in certain sub-populations, including men who have sex with

Correspondence: Ricardo Fuertes GAT Avenida Gomes Pereira, 98 - $4^{\circ}$ andar 1500-332 Lisboa, Portugal Tel: +351 210967 826; Fax: +351 210 938 216; e-mail: ricardo.fuertes@gatportugal.org men (MSM), migrants, injecting drug users and sex workers [1]. Although injecting drug has been an important driver of the HIV epidemic in Portugal, cases associated with injection of drugs have strongly declined over the past decade and the proportion of cases attributed to sex between men has increased. For the 776 new cases diagnosed and notified in 2012 in Portugal, 63.1\% ( $=$ 490) 
were attributed to heterosexual transmission, 24.1\% ( $\mathrm{n}=$ $187)$ to sex between men and $10.2 \%(n=79)$ to injecting drug use [2].

HIV testing has been a cornerstone of AIDS prevention strategies, as early diagnosis and treatment have both individual and public health benefits. Most-at-risk populations have been specifically targeted, and it has been recommend that MSM should be tested annually, or more often depending on sexual behavior [1].

In Portugal, HIV testing is available at hospitals, primary care centers, tuberculosis and drug treatment centres, and private laboratories. Free anonymous HIV testing is also available through outreach teams, and at 18 designated testing centres, one in each health region. In addition, Lisbon has established a community, peer-based site that provides free anonymous counseling and testing specifically targeted at MSM.

Information about HIV testing among MSM in Portugal is scarce. Our objective was to describe HIV testing behavior and context in a large sample of MSM participating in the European MSM Internet Survey (EMIS).

\section{Methods}

EMIS methods have been described in detail elsewhere [3]. In brief, EMIS was a joint project of academic, governmental and non-governmental partners from 38 countries in Europe to simultaneously run an online survey in 25 different languages during summer 2010. EMIS was approved by the Research Ethics Committee of the University of Portsmouth, UK(REC application number 08/09:21).

Data for the Portuguese sample were extracted and those for 5187 participants were analysed. Associations were examined using odds ratios (aOR) and 95\% confidence intervals (95\%CI), crude and adjusted for age, country of birth, educational level, sexual orientation disclosure, and UAI (unprotected anal intercourse) in the previous 12 months.

\section{Results}

The proportion of EMIS participants in Portugal tested for HIV infection during their lifetime was 72\% ( $\mathrm{n}=3723$ ), and $65 \%$ of those without known infection had tested for HIV in the last 12 months. Among those ever tested, 11\% were diagnosed with HIV. Among recently tested men who remained HIV negative at the time of survey, family doctors at National Health Service primary care centres were the most common providers of testing (37\%), followed by community HIV testing service (19\%), hospitals (17\%), private practice (15\%) and blood banks while donating blood (7\%). A high proportion (90\%) were satisfied with the way the testing service maintained confidentiality and ensured respectful treatment (92\%) at their last HIV test. However, only about half were satisfied with the counselling received and 38\% reported not having received any counselling.

Ever testing was most frequent among men aged 35-44 years and least frequent among those under 25 (83\% vs. 52\%, respectively; $P<0.001)$. However, among those ever tested, previous year testing was most frequent in men under 25 (77\%). Compared to those who had never been tested, men who had ever performed an HIV test had higher educational level, identified themselves as gay/homosexual more frequently and were out to most acquaintances (Table 1). Also, HIV testing was more frequent among participants living with a male partner (83\% vs. 70\% in those living with a female partner and 61\% in those living with others).

Those who reported unprotected anal intercourse (UAI) with a partner of unknown or serodiscordant HIV status in the previous 12 months, were significantly less likely to have ever taken an HIV test (aOR 0.38, 95\% CI 0.33-0.44). Men who had visited sex venues (aOR 2.26, 95\% CI 1.942.63 ) or had sex abroad in the previous year (aOR 2.20, 95\% CI 1.90-2.56) were more likely to have ever had a test. The odds of having taken at least one HIV test significantly increased with the number of sexual partners in the previous 12 months: those who had had one or between two and five partners were approximately four times more likely to have had an HIV test than those who reported no sexual partners in that period and the odds of being tested increased with the number of partners (6-10 partners, aOR 6.40, 95\% CI 4.77-8.58; above 10 partners aOR 9.51, 95\% CI 7.05-12.83). Previous testing was more commonly reported by men who reported the use of injection drugs at least once during their lifetime (aOR 1.54, 95\% CI 1.08-2.20).

Among those who never tested ( $n=1421)$, about twothirds (41\%) reported UAI with a partner of unknown or serodiscordant status in the previous 12 months and $57 \%$ had had at least five different sexual partners in the same period. The majority (81\%) of those who had never been tested were, however, very or quite confident that they could get a test for HIV if they wanted to. Among men who tested negative in their last HIV test ( $n=3244), 22 \%$ reported UAI with a partner of unknown or serodiscordant HIV status in the previous 12 months.

About half of those who were diagnosed with HIV (total 405) knew their CD4 count at diagnosis, and of those 37\% were diagnosed late (defined as having CD4 count $<350$ cells $/ \mu \mathrm{L})$. Linkage to care among men with diagnosed HIV was high: 97\% had visited a health professional in the previous six months. Seventy-two percent were currently 
Table 1 HIV testing uptake in MSM in Portugal according to participants' characteristics ( $n=5187)$ : results from EMIS, 2010

\begin{tabular}{|c|c|c|c|c|}
\hline & $\begin{array}{l}\text { Ever tested } \\
n(\%)\end{array}$ & $\begin{array}{l}\text { Never tested } \\
n(\%)\end{array}$ & OR $[95 \% \mathrm{Cl}]$ & aOR $[95 \% \mathrm{Cl}]$ \\
\hline \multicolumn{5}{|l|}{ Age (years) } \\
\hline$<25$ & $755(20.3)$ & $690(48.6)$ & 1 & 1 \\
\hline $25-39$ & $2067(55.5)$ & $532(37.4)$ & $3.55[3.08-4.09]$ & $3.28[2.83-3.81]$ \\
\hline$\geq 40$ & $901(24.2)$ & $199(14.0)$ & $4.14[3.44-4.98]$ & $4.25[3.48-5.19]$ \\
\hline \multicolumn{5}{|l|}{ Educational level } \\
\hline Low/medium & $1434(38.9)$ & $764(54.5)$ & 1 & 1 \\
\hline High & $2256(61.1)$ & $637(45.5)$ & $1.89[1.67-2.14]$ & $1.57[1.37-1.80]$ \\
\hline \multicolumn{5}{|l|}{ Sexual identity } \\
\hline Bisexual/Other & $971(26.2)$ & $577(40.7)$ & 1 & 1 \\
\hline Gay/Homosexual & 2741 (73.8) & $840(59.3)$ & $1.94[1.70-2.20]$ & $2.02[1.75-2.33]$ \\
\hline \multicolumn{5}{|c|}{ Sexual orientation disclosure } \\
\hline \multicolumn{5}{|l|}{ Disclosure } \\
\hline 'Out of the closet' & $1592(43.0)$ & $370(26.2)$ & 1 & 1 \\
\hline 'In the closet' & $2114(57.0)$ & $1044(73.8)$ & $0.47[0.41-0.54]$ & $0.51[0.44-0.59]$ \\
\hline \multicolumn{5}{|l|}{ UAI in last 12 months } \\
\hline No & $2791(76.7)$ & $826(59.0)$ & 1 & 1 \\
\hline Yes & $847(23.3)$ & $574(41.0)$ & $0.44[0.38-0.50]$ & $0.38[0.33-0.44]$ \\
\hline \multicolumn{5}{|c|}{ Sexual partners in last 12 months } \\
\hline 0 & $155(4.2)$ & $236(16.8)$ & 1 & 1 \\
\hline 1 & $775(21.1)$ & $295(21.1)$ & $4.00[3.18-5.10]$ & $3.95[3.02-5.17]$ \\
\hline $2-5$ & $1254(34.2)$ & $497(35.5)$ & $3.84[3.06-4.82]$ & $4.70[3.64-6.08]$ \\
\hline $6-10$ & $625(17.0)$ & $198(14.1)$ & $4.81[3.71-6.22]$ & $6.40[4.77-8.58]$ \\
\hline$>10$ & $858(23.4)$ & $175(12.5)$ & $7.46[5.76-9.68]$ & 9.51 [7.05-12.83] \\
\hline \multicolumn{5}{|l|}{ Sex abroad } \\
\hline No & $1710(46.4)$ & $1020(72.4)$ & 1 & 1 \\
\hline Yes & $1971(53.6)$ & $388(27.6)$ & $3.03[2.65-3.46]$ & $2.20[1.90-2.56]$ \\
\hline \multicolumn{5}{|l|}{ Sex venues } \\
\hline Never & $978(26.4)$ & $714(50.5)$ & 1 & 1 \\
\hline At least once & $2734(73.6)$ & $700(49.5)$ & $2.85[2.51-3.24]$ & $2.26[1.94-2.63]$ \\
\hline \multicolumn{5}{|c|}{ Syphilis history (self-reported) } \\
\hline No & $3269(88.5)$ & $1376(97.3)$ & 1 & 1 \\
\hline Yes & $425(11.5)$ & $38(2.7)$ & $4.71[3.36-6.60]$ & $4.42[3.05-6.41]$ \\
\hline \multicolumn{5}{|l|}{ Injecting drug use } \\
\hline Never & 3480 (94.8) & $1343(96.5)$ & 1 & 1 \\
\hline At least once & $189(5.2)$ & $49(3.5)$ & 1.49 [1.08-2.05] & $1.54[1.08-2.20]$ \\
\hline
\end{tabular}

aOR: Adjusted for age, country of birth, educational level, sexual orientation disclosure and UAI in the last 12 months: unprotected anal intercourse in the last 12 months.

aOR, adjusted odds ratio; $\mathrm{Cl}$, confidence interval; $\mathrm{OR}$, odds ratio; $\mathrm{UAl}$, unprotected anal intercourse.

on antiretroviral therapy (ART) (after excluding 27\% who did not disclose therapy): those treated included 56\% of patients with a CD4 count $>350$ cells/ $\mu \mathrm{L}$ at diagnosis and $71 \%$ of late presenters. Overall, 58\% reported having an undetectable viral load. More than one third (38\%) of those infected who had detectable or unknown/undisclosed viral load reported at least on episode of UAI with a partner of unknown or serodiscordant HIV status in the last 12 months.

\section{Discussion}

The increased incidence of HIV in gay communities has been documented in many other countries, and the paradoxical increase in HIV incidence among MSM over recent years despite increased ART coverage has been explained by an increase in condomless sex [4,5]. In our sample of
MSM, UAI in the previous year was reported by 22\% of those who tested HIV negative and by $41 \%$ of those who had never been tested, which means that the number of men at risk as well as non-diagnosed HIV infections may be substantial. There is evidence that new infections are mainly the result of transmission from people who are yet to be diagnosed [4], so emphasis on HIV testing is of paramount importance.

Perception of structural barriers to testing in this sample did not seem to be determinant, as $81 \%$ of those never tested were confident that they could take a test. Previously, a low perceived risk of infection was the single most important barrier (reported by $80 \%$ ) to testing found in a sample of 301 participants diagnosed between 2005 and 2008 in Portugal (18\% were MSM) [6] but further studies are needed to address this question in this specific population. 
Family doctors, hospitals and community HIV testing services were the most common providers of testing, but the proportion of MSM who used blood banks for HIV testing was high (7\%), even though the current policy in Portugal is to screen MSM out of blood donations. As for contextual factors associated with HIV testing, while confidentiality and respect were considered satisfactory, counselling was considered satisfactory by only half of the participants and more than one third did not receive any counselling at their last test, highlighting the need to reinforce the importance of counselling and its quality among health professionals and social workers. We could not assess the extent to which MSM voluntarily opted out of counselling.

HIV testing is required to ensure that infected individuals enter clinical care and receive appropriate treatment in a timely fashion. About three-quarters of our sample had taken at least one HIV test during their lifetime, and 11\% were diagnosed with HIV infection. Linkage to care was almost universal (94\%) but was not completely predictive of ART coverage or viral load undetectability.

In recent years there has been a renewed emphasis on testing with the focus on treatment as prevention [7] but this strategy will only work if infected people are diagnosed earlier and indeed treated effectively. In our sample, over one third of those infected who had detectable or unknown/ undisclosed viral load reported at least one episode of UAI with a partner of unknown or serodiscordant HIV status in the last 12 months. These findings stress the need to clearly communicate that even someone on treatment might still be infectious and thus consistent condom use should be strongly encouraged for most MSM, even in times of broad access to and uptake of ART.

Limitations: Although the sample was large, representing 5187 MSM in Portugal, it was non-random.

The EMIS data are likely to be biased towards those who are better educated and internet-literate, and probably more familiar with the gay subculture. Nonetheless, despite the self-selection and recall biases, this is the largest sample of MSM ever studied in Portugal.

The analysis of this large sample of MSM in Portugal suggests that actual interventions should focus on: improving testing uptake and counselling, especially in primary care (the most common testing setting); increasing treatment coverage; achieving and maintaining an undetectable viral load; and intensifying prevention efforts focussed on consistent condom use.

\section{Acknowledgements}

We would like to acknowledge the scientists who organised and conducted EMIS between 2009 and 2011: Axel J. Schmidt (project co-ordination); Ulrich Marcus (project ini- tiation and supervision); Peter Weatherburn (promotion co-ordination); Ford Hickson and David Reid (Technical implementation); Harm J. Hospers (questionnaire drafting).

Funding: EMIS was funded by a grant of the European Commission under the EU Health Programme 2008-2013. Further funding was received from CEEISCat (Centre d'Estudis Epidemiològics sobre les ITS/HIV/SIDA de Catalunya, Spain); Department of Health for England (UK); Maastricht University (The Netherlands); Regione del Veneto (Italy); and Robert Koch Institute (Germany). Further funding for the participation of men in specific countries was provided by: German Ministry of Health, for Ukraine and Moldova; Finnish Ministry of Health, for Finland; Norwegian Institute of Public Health, for Norway; Swedish Board of Health and Welfare, for Sweden; and Bundeszentrale für gesundheitliche Aufklärung (BZgA), for Germany. Scientific co-ordination: Robert Koch Institute (Germany); Administrative co-ordination: GIZ-Gesellschaft für Internationale Zusammenarbeit (Germany); Technical Implementation: Sigma Research, London School of Hygiene \& Tropical Medicine (UK); Questionnaire drafting: University College, Maastricht (The Netherlands).

Conflicts of interest: All authors state that they have no conflicts of interest to disclose.

\section{References}

1 European Centre for Disease Prevention and Control. HIV Testing: Increasing Uptake and Effectiveness in the European Union. Stockholm, ECDC, 2010.

2 National Institute of Health Dr. Ricardo Jorge, Department of Infectious Diseases. HIV/AIDS: the situation in Portugal December 31, 2012. INSA; 2013.

3 Weatherburn P, Schmidt AJ, Hickson F et al. and the EMIS Network. The European Men-who-have-sex-with-men Internet Survey (EMIS): design and methods. Sex Res Soc Policy in press; 2013. doi: 10.1007/s13178-013-0119-4.

4 Phillips AN, Cambiano V, Nakagawa F, Brown AE, Lampe F et al. Increased HIV incidence in men who have sex with men despite high levels of ART-induced viral suppression: analysis of an extensively documented epidemic. PLoS ONE 2013; 8: e55312. doi:10.1371/journal.pone.0055312.

5 De Wit JBF, Prestage GP, Duffin IR. Gay men: current challenges and emerging approaches in HIV prevention. 21: 3-4. NSW Public Health Bull 2010.

6 Carvalho C. HIV test: access, practices and barriers. HIV Conference 2009. Lisbon. Acção e Tratamentos n²3, GAT.

7 World Health Organization. Scaling up HIV Testing and Counseling in the WHO European Region - As an Essential Component of Efforts to Achieve Universal Access to HIV Prevention, Treatment, Care and Support. Policy Framework. Geneva, World Health Organization, 2010. 